\title{
TCOM AMERICA LATINA \\ Estado del arte de la divulgación de la ciencia en América Latina
}

\section{Luisa Massarani}

Resumen

En este artículo, tenemos como objetivo presentar el estado del arte de la divulgación de la ciencia en América Latina con base en una revisión de publicaciones realizadas en el ámbito de la Red de Popularización de la Ciencia y la Tecnología en América Latina y el Caribe (RedPOP), entre 2014 y 2017. Con dichas publicaciones, se realizó un diagnóstico en el campo en la región. Incluimos aquí aspectos relacionados a las actividades prácticas, a la investigación, a la política científica y a la capacitación en divulgación de la ciencia.

Palabras clave

DOI

Introducción
Historia de la divulgación de la ciencia; Divulgación de la ciencia en los países en desarrollo

https://doi.org/10.22323/3.01010201

Fecha de recepción: 3 de septiembre de 2018

Fecha de aceptación: 27 de septiembre de 2018

Fecha de publicación: 21 de noviembre de 2018

El origen de la divulgación de la ciencia como práctica se confunde con el propio proceso de creación de la ciencia: ya en el siglo XVII, Galileo se esforzaba para divulgar sus hallazgos en física y astronomía [Massarani y De Castro Moreira, 2004]. En los siglos siguientes, las actividades divulgativas continuaron en expansión y, hacia el siglo XIX, se intensificaron por todo el mundo. En América Latina, como ejemplo de lo que ocurrió en otras partes del mundo, los propios investigadores divulgaban descubrimientos con el propósito de afirmar su legitimidad profesional y fortalecer alianzas con los pares científicos e instituciones importantes [Massarani y De Castro Moreira, 2004].

El surgimiento de la divulgación de la ciencia como campo de estudio, sin embargo, data de un período mucho más reciente. Trench y Bucchi [2010] afirman que la divulgación de la ciencia se definió como un campo de estudio durante los últimos 20-30 años, en una intersección entre "ciencias de la educación, estudios sociales de la ciencia, estudios de medios masivos de comunicación, museología y muchas actividades académicas y profesionales bien establecidas" [Trench 
y Bucchi, 2010, p. 3]. En América Latina, el campo académico también inició aunque lentamente - en la década de los ochentas.

Sin embargo, es importante recordar que América Latina abarca una gran extensión territorial que va desde México hasta la Tierra de Fuego, que representa casi el $13 \%$ de la superficie terrestre y tiene una gran diversidad cultural.

Cambre [2015, p. 42] afirma que, en América Latina, "si bien es cierto que hay muchos lazos en común que los unen, no es menos cierto que también existen entre ellos diferencias culturales que les otorgan un distintivo propio". La autora apunta la existencia de una variedad étnica en la región conformada por los descendientes de los pueblos nativos, los conquistadores europeos, los esclavos provenientes de África, las corrientes migratorias posteriores (siglos XIX y XX) y el mestizaje producto de la unión de las diferentes poblaciones, siendo que cada uno de estos grupos se ha integrado en las sociedades de diversas maneras y con distinto peso específico en cada país.

Llamando la atención para el hecho de que la pobreza y la exclusión cultural son una realidad en un porcentaje alto de la población de estos países, Cambre afirma:

\footnotetext{
"Si algo tenemos en común los países latinoamericanos son los ciclos de crisis económicas, sociales y políticas. Estos devenires hicieron que ciencia y tecnología no estuvieran en la prioridad de las agendas políticas de nuestros gobernantes al mismo tiempo que sí lo estaban en países del primer mundo." [Cambre, 2015, p. 42]
}

En este contexto de diversidad en la región, los distintos países también tienen historias diferentes con respecto a la divulgación de la ciencia, cuyos registros de actividades en la región existen desde hace al menos dos siglos [ver, por ejemplo, Moreira y Massarani, 2002; Sánchez-Mora y col., 2015; Fog, 2004]. Sin embargo, los caminos que llevaran al diálogo - en mayor o menor medida - entre ciencia y sociedad también pueden ser diferentes. Massarani, Aguirre y col. [2015], por ejemplo, analizaron las trayectorias recorridas en países de la región que tienen una cultura científica importante como Argentina, Brasil, Colombia y México.

En este artículo, tenemos como objetivo presentar el estado del arte de la divulgación de la ciencia en América Latina, incluyendo aspectos relacionados a las actividades prácticas, a la investigación, a la política científica y a la capacitación en divulgación de la ciencia. Para ello, hacemos una revisión de publicaciones realizadas en el ámbito de la Red de Popularización de la Ciencia y la Tecnología en América Latina y el Caribe (RedPOP), entre 2014 y 2017, en colaboración con otras organizaciones, buscando realizar un diagnóstico del campo en la región, que tuvieron el apoyo del entonces Ministerio da Ciencia, Tecnología, Innovaciones y Comunicaciones (MCTIC) de Brasil, a través del Consejo Nacional de Desarrollo Científico y Tecnológico (CNPq). Aunque las publicaciones impulsadas por RedPOP no son exhaustivas, permiten presentar un primer panorama general del campo. 
Los distintos términos
Fernandez, Bello y Massarani [2015] apuntan que la primera dificultad metodológica al momento de emprender una investigación en esta materia es la dispersión de denominaciones y conceptos, la falta de una definición internacionalmente reconocida y de consenso académico acerca de su abordaje. Corroborando dicha afirmación, Rocha y Massarani [2017], en un análisis de 609 artículos académicos sobre divulgación de la ciencia escritos por latinoamericanos y/o con estudios realizados en América Latina, observaron en ellos que tampoco hay consenso sobre la definición, ni claridad sobre eventuales similitudes y diferencias, entre los distintos términos y conceptos usados en el campo. También observaron una diversidad de términos utilizados para el campo, como muestra la Tabla 1 abajo.

Tabla 1. Términos utilizados para definir divulgación de la ciencia y el porcentaje de artículos que hace uso de cada término. El total supera el $100 \%$ ya que un mismo artículo, a veces, usaba más de un término. Fuente: Rocha y Massarani [2017].

\begin{tabular}{|l|r|}
\hline Términos & Porcentaje de artículos \\
\hline Divulgación de la ciencia & $62,8 \%$ \\
\hline Comunicación de la ciencia & $20,3 \%$ \\
\hline Educación no formal en ciencia & $18,1 \%$ \\
\hline Popularización de la ciencia & $15,6 \%$ \\
\hline Alfabetización científica & $11,6 \%$ \\
\hline Comunicación pública de la ciencia & $6,9 \%$ \\
\hline Percepción social de la ciencia & $3,3 \%$ \\
\hline Democratización de la ciencia & $0,4 \%$ \\
\hline Apropiación social del conocimiento científico & $0,5 \%$ \\
\hline
\end{tabular}

La Figura 1 permite ver la presencia dinámica de los términos a lo largo del periodo de estudio de Rocha y Massarani [2017], cuya recolección tuvo lugar entre marzo y septiembre de 2016. Los artículos publicados en el último trimestre no fueron considerados en el análisis. Así y para evitar que el conjunto de artículos del año 2016 quedara desfasado, Rocha, Massarani y Pedersoli [2017] realizaron una proyección de las publicaciones para los meses de octubre, noviembre y diciembre en base en los nueve meses anteriores. Para identificar qué términos de esos artículos serían utilizados, se aplicaron los porcentajes de artículos que utilizaron cada término durante los nueve meses anteriores. En este escenario, las autoras observaron que el término divulgación de la ciencia disminuyó desde 2014, mientras que comunicación y la popularización de la ciencia crecieron.

Al comparar los datos con los arrojados por Google Ngram Viewer, Rocha, Massarani y Pedersoli [2017] observaron algunas coincidencias. A pesar de algunas limitaciones de la herramienta referidas a características propias de la base de datos desde la que rescata información ("varios libros" según allí se los identifica), los resultados sirven a modo de referencia para mostrar algunas tendencias (Figura 2). En este caso también se observó un aumento de los términos popularización y comunicación de la ciencia, al mismo tiempo que una curva ascendente en la utilización de la categoría divulgación de la ciencia. De hecho, ésta apareció en ese relevamiento como el término más utilizado en los libros que componen la base de búsqueda. 


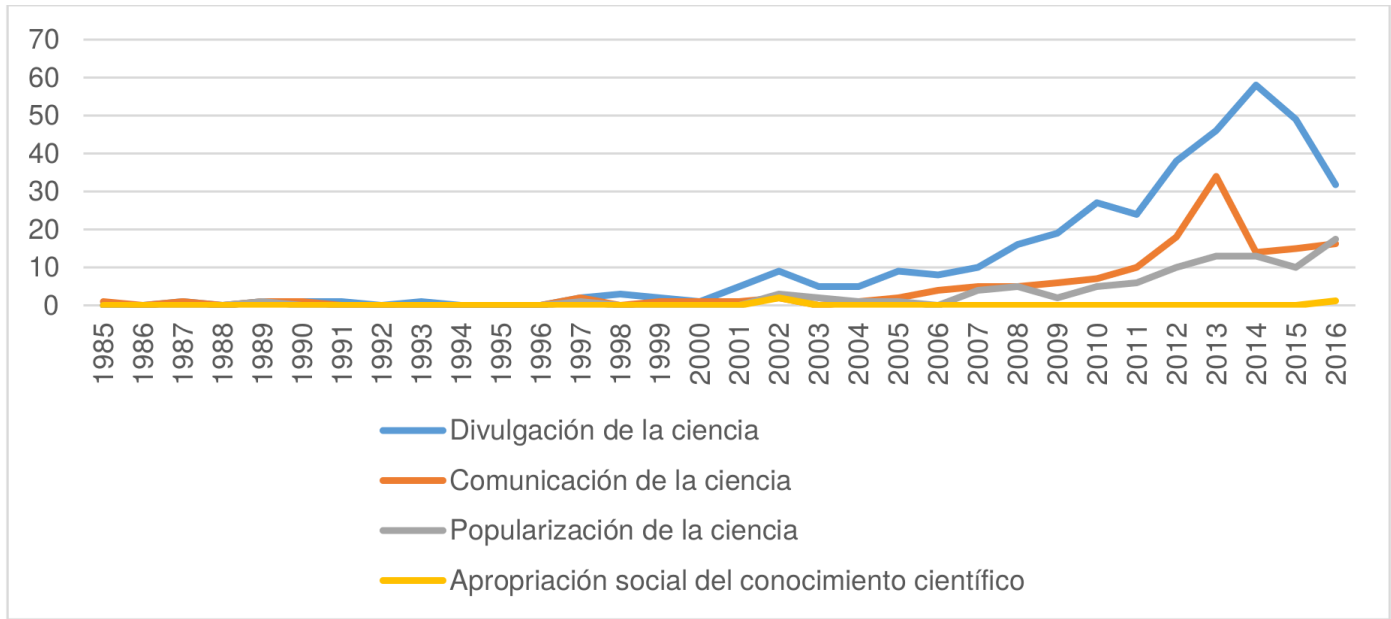

Figura 1. Frecuencia de los términos utilizados en los artículos de acuerdo al año de publicación. Fuente: Rocha, Massarani y Pedersoli [2017].

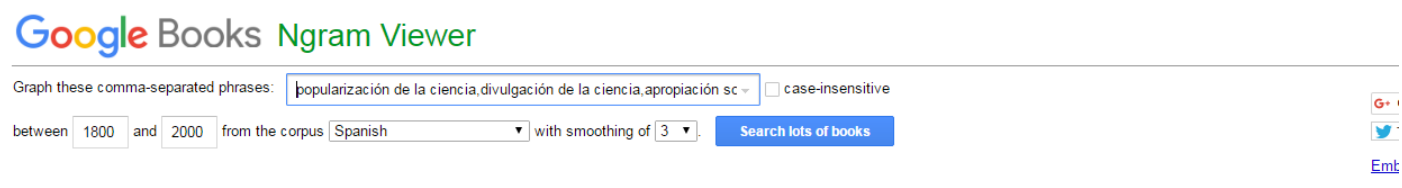

\section{Práctica en divulgación de la ciencia}

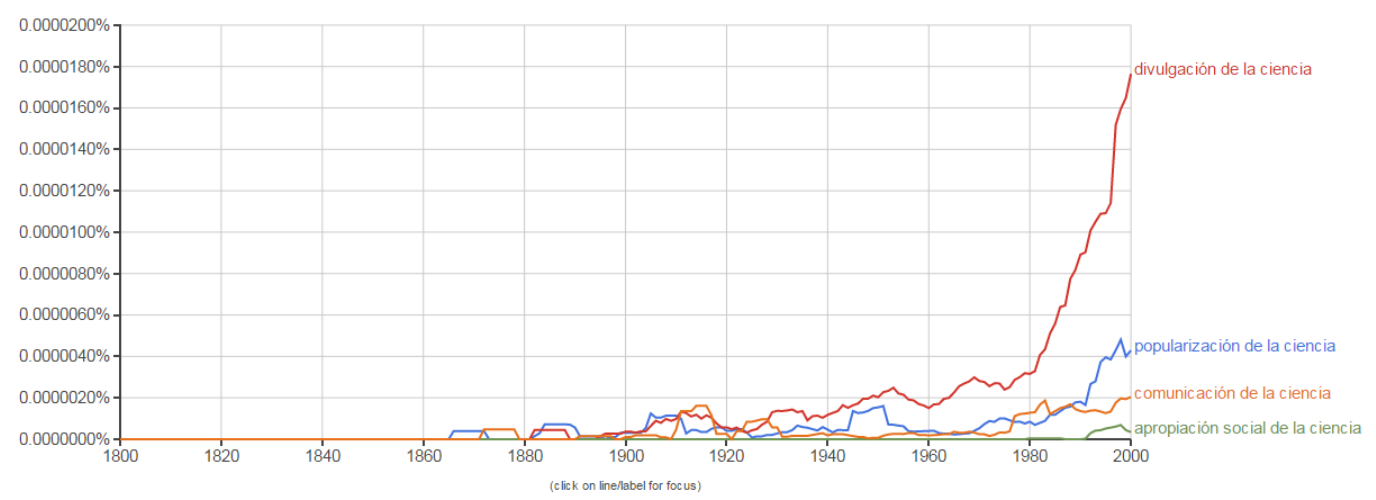

Figura 2. Palabras claves buscadas: popularización de la ciencia, divulgación de la ciencia, apropiación social de la ciencia, comunicación de la ciencia. Fuente: Google NgramViezver [https://books.google.com/ngrams]. ${ }^{1}$

Patiño, Padilla y Massarani [2017] identificaron una diversidad de actores sociales, tanto institucionales como individuales, que han realizado esfuerzos para divulgar la ciencia y la tecnología en los países de América Latina, como seguir:

- Universidades e instituciones de educación superior que cuentan con programas o acciones de divulgación de la ciencia.

- Institutos y centros de investigación que, además de sus labores de investigación y formación de capital humano, desarrollan acciones de divulgación científica.

\footnotetext{
${ }^{1}$ Es importante comentar que aunque cultura científica no tuvo una presencia importante en el corpus del estudio de Rocha y Massarani [2017] y de Rocha, Massarani y Pedersoli [2017] fue el término más utilizado al largo del siglo XX, con un pico en los años 1920 y un descenso posterior. En los años 1940 hubo también un incremento del uso de este término, con posterior descenso y posterior incremento en los años 1990 y 2000.
} 
- Dependencias públicas o gubernamentales que realizan divulgación científica como parte de sus funciones naturales, en los campos disciplinares que les son propios [salud, medio ambiente, etc.].

- Recintos que ofrecen al público experiencias con el conocimiento científico y tecnológico, y que cuentan con programas de divulgación y educación no formal: museos de antropología y de ciencias naturales, museos de ciencia, centros interactivos de ciencias, zoológicos, acuarios, jardines botánicos, planetarios, etc.

- Medios de comunicación que se enfocan total o parcialmente en la divulgación de la ciencia [radio, televisión, prensa escrita, revistas, redes sociales y sitios de Internet].

- Grupos organizados [privados y públicos] de divulgadores.

- Asociaciones y redes de organizaciones y de profesionales de la divulgación.

Para comprender mejor la práctica de la divulgación de la ciencia y los desafíos enfrentados por las organizaciones que las realizan, Patiño, Padilla y Massarani [2017] realizaron un estudio diagnóstico, a través de una encuesta en línea, a la cual respondieron 123 instituciones con programas de divulgación de la ciencia en la región. El levantamiento de información fue realizado en un lapso de tres meses (marzo a mayo de 2016). Entre las consideraciones conclusivas, los autores afirman que las actividades de divulgación de la ciencia en la región, de una manera general, están aún lejos de ser sistemáticas y cotidianas en la vida de los latinoamericanos. Vale señalar, también, los siguientes resultados del estudio:

- Las estrategias y los medios de divulgación de la ciencia utilizados son variados - de acuerdo con lo reportado por las instituciones participantes del estudio - predominando los eventos y programas (tales como conferencias, talleres, muestras de exhibiciones y prototipos, ferias de ciencias, etc.).

- Ha ganado mucha participación la divulgación de la ciencia a través de medios digitales e Internet, recursos que el avance tecnológico ha facilitado que estén a disposición de los divulgadores.

- Gran parte de las acciones de divulgación de la ciencia se enfocan en el sector escolarizado (estudiantes y docentes), que constituye un público más definido y en general más accesible para los divulgadores, que otros sectores de la sociedad. El segmento de adultos podría considerarse sub-atendido.

- En general las instituciones latinoamericanas que realizan divulgación manifiestan algunas debilidades estructurales, organizacionales y de recursos para llevar a cabo esta tarea, incluso con muchas instituciones que no poseen un plan de acción ni presupuesto para trabajar.

- Es todavía un campo muy basado en acciones de personas que son voluntarias.

- La documentación, el registro y la evaluación de impacto de las actividades realizadas es escasa en la región. 
Tabla 2. Visita a espacios de ciencia de acuerdo con los que respondieron a encuestas nacionales y regionales sobre percepción pública de la ciencia y la tecnología en Iberoamérica. Fuente: Polino [2015].

\begin{tabular}{|l|l|r|}
\hline \multicolumn{2}{|l|}{ Dígame si durante este año hizo alguna de estas actividades: } & Sí \\
\hline \multirow{2}{*}{$\begin{array}{l}\text { Argentina } \\
\text { (2012) }\end{array}$} & Visitar un museo de ciencia y tecnología. & $17,7 \%$ \\
& Visitar un zoológico, botánico o acuario. & $26,3 \%$ \\
& Visitar un parque nacional o reserva natural. & $24,7 \%$ \\
& Asistir a la Semana Nacional de la Ciencia. & $6,1 \%$ \\
\hline \multirow{3}{*}{ Brasil } & Visitar un centro o museo de ciencia y tecnología. & $12,3 \%$ \\
$\mathbf{( 2 0 1 5 )}$ & Visitar un zoológico. & $26,1 \%$ \\
& Visitar un jardín botánico o parque ambiental. & $31,3 \%$ \\
& Asistir a la Semana Nacional de la Ciencia. & $8 \%$ \\
\hline \multirow{4}{*}{ Colombia } & Visitar un museo de ciencia y tecnología. & $19,7 \%$ \\
(2012) & Visitar un zoológico o acuario. & $40,1 \%$ \\
& Visitar un parque natural. & $48,4 \%$ \\
\hline \multirow{3}{*}{ México } & Asistir a la Semana Nacional de la Ciencia. & $11,3 \%$ \\
\hline (2011) & Visitar un museo de ciencia y tecnología. & $15,6 \%$ \\
& Visitar un zoológico o acuario. & $43,4 \%$ \\
& Visitar un parque nacional o reserva natural. & - \\
\hline \multirow{2}{*}{ Uruguay } & Asistir a la Semana Nacional de la Ciencia. & $8,1 \%$ \\
(2011) & Visitar un museo de ciencia y tecnología. & $22 \%$ \\
& Visitar un zoológico, botánico, acuario, reserva o planetario. & $30 \%$ \\
& Asistir a la Semana Nacional de la Ciencia. & $12 \%$ \\
\hline
\end{tabular}

Los museos de ciencia
Entre los actores sociales y espacios que realizan actividades de divulgación de la ciencia, vale destacar los museos de ciencia que, aunque creados especialmente a partir de los años 1990 en América Latina, han ganado importancia social en la región. Aunque bajo el riesgo de supervalorarlos con respecto a otros actores sociales que también son importantes, a continuación presentaremos información sobre ellos, justamente porque fueron el blanco de uno de los relevamientos liderados por RedPOP.

La iniciativa resultó en una Guía de Museos de Ciencia de América Latina y Caribe [Massarani, Aguirre y col., 2015], en la cual el concepto de museo de ciencia fue amplio, incluyendo 468 centros de ciencia interactivos, museos de historia natural, planetarios, zoológicos, acuarios, entre otros. Es importante decir que este número no es exhaustivo, visto que solo fueron incluidos en la publicación las instituciones que accedieron a enviar su información. Por un lado, observamos una concentración de los espacios en pocos países - como Brasil, que concentra 272 espacios descritos en la guía - por otro lado, observamos que 22 países de la región están presentes. El porcentaje de la sociedad que visita estos espacios varía de país a país y es todavía reducido, llamando la atención para una exclusión social importante que todavía existe en la región - aunque creciente, como señala el caso de Brasil que en 10 años triplicó el número de visitantes. La Tabla 2, de Polino [2015], es ilustrativa de las diferencias entre países, consolidada con datos de encuestas nacionales de los países: 
Un aspecto discutido por Cambre [2015] se refiere a la medida en la que los museos de ciencia de América Latina tienen una personalidad propia, un color regional. La autora defiende que se pueden diferenciar dos etapas o periodos en el diseño, la programación y la construcción de los centros interactivos en Latinoamérica: antes y después del año 2000. En su opinión, en el primer periodo se encuentran los pioneros en sus respectivos países, y ahí la diversidad cultural y la impronta local no son una característica marcada en sus comienzos. Ella afirma:

\begin{abstract}
Hay dos factores principales que incidieron, uno es que la ciencia y sus principios así como los desarrollos tecnológicos son transversales a cualquier sociedad por lo tanto los primeros centros interactivos eran en general museos "sin personalidad local", fenómeno que se dio en todo el mundo. El otro factor fue la tendencia a trasladar y copiar los modelos exitosos en el mundo por falta de recursos humanos especializados y experiencia en la nueva modalidad museográfica que se estaba imponiendo. [Cambre, 2015, p. 44]
\end{abstract}

Sin embargo, Cambre defiende que en la "nueva etapa los museos latinoamericanos han ido interpretando la realidad de la sociedad en la cual se encuentran y no solo han incorporado lo local a los temas globales sino también han desarrollado estrategias y modelos museográficos que son ejemplo mundialmente" [Cambre, 2015, p. 44].

Otro aspecto relacionado a museos de ciencia - aunque por supuesto no limitado a ellos, pero si a toda la sociedad - es el tema de la accesibilidad. En foros ha sido consenso la importancia de incrementar la accesibilidad en museos de ciencia y programas de divulgación científica, pero la realidad enseña los desafíos importantes de poner este deseo en práctica: en el estudio mencionado anteriormente realizado por Patiño, Padilla y Massarani [2017] con 123 instituciones con programas de divulgación de la ciencia en la región solo aproximadamente el $37 \%$ de ellas afirmaron que cuentan con algún programa enfocado a personas con discapacidad, siendo las que tienen discapacidades visual y de movilidad las más atendidas, con el $13.6 \%$ y el $7.3 \%$ respectivamente. La Guía de Museos y Centros de Ciencias Accesibles de América Latina y el Caribe, realizado por el Grupo Museos y Centros de Ciencias Acesibles [MCCAC] y la RedPOP y colaboradores [Norberto Rocha y col., 2017], es ilustrativo de la situación: se apuntaron 110 espacios, de diez países de América Latina y el Caribe, que tienen niveles diferentes de acciones de accesibilidad, entre los 468 registrados en la Guía de Museos de Ciencia de América Latina y Caribe.

Política científica en divulgación de la ciencia
Otro estudio realizado en el contexto de comprender la divulgación de la ciencia en la región - y que sería importante ampliar para otras regiones del globo terrestre - es un diagnóstico de los países con marcos legales y/o normativas jurídicas específicas destinadas al sector en los distintos países de la región [Fernandez, Bello y Massarani, 2015; Fernandez, Bello y Massarani, 2016]. Según los autores, en los últimos tiempos, distintos países han empezado a incorporar en sus marcos legales referencias a la cultura científica o han establecido capítulos y normativas específicas en la materia, entre ellos 14 países de la región: Argentina, Bolivia, Brasil, Chile, Colombia, Costa Rica, Ecuador, El Salvador, Guatemala, México, Panamá, Paraguay, Perú y Uruguay. 
Los autores apuntan que hay distintos enfoques en dicha incorporación.

Hay países en que la única mención en la legislación es la identificación del tema que se halla en las leyes que definen los objetivos y visiones de los organismos nacionales de ciencia y tecnología, o en las que establecen un organismos ad hoc, como parte integrante del sistema Nacional de Ciencia y Tecnología (como pasa en Bolivia, Chile, Colombia, El Salvador, Ecuador, Panamá y Uruguay).

En otro grupo de países existen leyes (como las de Argentina, Costa Rica, Guatemala, México, Paraguay y Perú) que dedican en sus respectivas legislaciones nacionales de CTI partes o capítulos enteros a esta temática o prevén explícitamente recursos para incentivar la promoción de una cultura científica (financiamiento de ferias, festivales o premios para incentivar la difusión de la ciencia y la tecnología, entre otros).

De manera particular, Brasil se encuentra en este ámbito. En 2004, fue creado el Departamento de Popularización y Difusión de la Ciencia y Tecnología en el entonces Ministerio de Ciencia y Tecnología, con la competencia de subsidiar la formulación y la implementación de políticas, programas y la definición de estrategias de popularización y la difusión de los conocimientos científicos y tecnológicos. Esto impulsó bastante la divulgación de la ciencia en el país con convocatorias para apoyar proyectos en el área por el Consejo Nacional de Desarrollo Científico y Tecnológica, que incluso creó un comité específico para el sector, después se difundió por varios estados, entre ellos Amazonas, Minas Gerais y Rio de Janeiro [Massarani y De Castro Moreira, 2016]. Sin embargo, con la fusión del ministerio de ciencia al de comunicación, la pérdida de estatus y los recortes de presupuesto para la ciencia en general - que hoy representa un tercio del presupuesto de 2010 — hay posibilidades reales de una pérdida importante en todo lo que fue estructurado a largo de los últimos años en el país.

De acuerdo con Fernandez, Bello y Massarani [2015] y Fernandez, Bello y Massarani [2016], en la mayoría de los países de la región, las principales actividades abocadas al desarrollo de una cultura científica son llevadas a cabo por programas nacionales. Sin embargo, la falta de creación de un programa específico en el país no debe ser asociada con la falta de acciones e instrumentos.

Los países de la región han puesto en marcha distintos tipos de instrumentos de cultura científica. Sobre la base de una serie de enfoques distintos identificados durante la investigación, los autores desarrollaron una clasificación de las iniciativas más frecuentes llevadas a cabo y de su público objetivo, en la Tabla 3 (ver la siguiente página).

Fernandez, Bello y Massarani [2015] y Fernandez, Bello y Massarani [2016] destacan que un aspecto que merece particular atención es la percepción pública de la ciencia y la tecnología [ver tambien Polino, 2015]. Según los autores, las encuestas se desarrollan con el objetivo de recopilar informaciones relevantes para la generación de indicadores para medir el conocimiento, entendimiento y actitud de la sociedad en lo que concierne las actividades científicas y tecnológicas.

Asimismo, apuntan a que sustenten políticas, instrumentos y acciones. En la región los distintos países han realizado encuestas en este sentido. 
En los últimos 15 años siempre más países han llevado a cabo encuestas nacionales sobre percepción pública de la ciencia y la tecnología. Hasta la fecha 11 países de la región han realizado encuestas nacionales, desde el 1987: Argentina, Brasil, Chile, Costa Rica, Colombia, Ecuador, El Salvador, República Dominicana, México, Panamá, Paraguay, Uruguay y Venezuela.

Tabla 3. Categorización de actividades de cultura científica. Fuente: Fernandez, Bello y Massarani [2016].

\begin{tabular}{|c|c|c|c|c|c|}
\hline $\begin{array}{l}\text { Eventos } \\
\text { de gran porte }\end{array}$ & Capacitaciones & $\begin{array}{l}\text { Premios y } \\
\text { Convocatorias }\end{array}$ & Medios masivos & $\begin{array}{l}\text { Museos y Centros } \\
\text { científicos y } \\
\text { tecnológicos }\end{array}$ & Otros \\
\hline $\begin{array}{l}\text { - Ferias } \\
\text { - Festival de } \\
\text { ciencia } \\
\text { - Muestras } \\
\text { - Semanas de la } \\
\text { Ciencia }\end{array}$ & $\begin{array}{l}\text { - Jornadas } \\
\text { - Talleres } \\
\text { - Encuentros } \\
\text { - Curso } \\
\text { - Seminario } \\
\text { - Otro tipo de } \\
\text { Capacitaciones }\end{array}$ & $\begin{array}{l}\text { - Concursos } \\
\text { - Premios } \\
\text { - Convocatorias } \\
\text { - Llamados públi- } \\
\text { cos } \\
\text { para apoyo finan- } \\
\text { ciero } \\
\text { [puede tratar- } \\
\text { se de llamados } \\
\text { dirigidos } \\
\text { a una categoría } \\
\text { en particular } \\
\text { (estudiantes, } \\
\text { periodistas etc.) o } \\
\text { a la sociedad en su } \\
\text { conjunto] }\end{array}$ & $\begin{array}{l}\text { - Sitios web } \\
\text { - Programas } \\
\text { de TV/ } \\
\text { - Planos de } \\
\text { periodismo } \\
\text { científico }\end{array}$ & $\begin{array}{l}\text { - Museos } \\
\text { - Museos } \\
\text { interactivos de } \\
\text { ciencia } \\
\text { - Museos de } \\
\text { historia natural } \\
\text { - Zoológicos } \\
\text { - Jardines } \\
\text { botánicos } \\
\text { - Planetarios } \\
\text { - Acuarios } \\
\text { - Iniciativas } \\
\text { itinerantes }\end{array}$ & $\begin{array}{l}\text { - Olimpiadas } \\
\text { - Clubes de } \\
\text { Ciencia } \\
\text { - Teatro } \\
\text { científico } \\
\text { - Campamentos } \\
\text { - Redes de } \\
\text { espacios de } \\
\text { educación } \\
\text { no formal de } \\
\text { divulgación } \\
\text { científica }\end{array}$ \\
\hline
\end{tabular}

Formación de divulgadores de la ciencia
Reynoso Haynes, Monterrosa y Macías [2015] señalan que hay tres generaciones de divulgadores de la ciencia en América Latina, con base en su formación. La primera generación corresponde a la de los pioneros que se formó en la práctica a base de ensayo y error. Sin embargo, muchos consideraron que esta preparación no era suficiente y que requerían una formación más sólida. Surgió así, de acuerdo con las autoras, una segunda generación de divulgadores de la ciencia. Como en aquella época todavía no existían programas especializados en divulgación de la ciencia, cursaron posgrados en áreas complementarias a su formación inicial como la pedagogía, la literatura, el periodismo y el cine.

De acuerdo con Reynoso Haynes, Monterrosa y Macías [2015], en la década de los años noventa, estas dos generaciones convencidas de que la divulgación de la ciencia es una labor que requiere de una formación especializada, conjuntaron sus experiencias y reflexiones para desarrollar programas con la finalidad de formar profesionales en el campo. Sin embargo, defienden los autores, no hay fórmulas únicas para diseñar el programa ideal para formar divulgadores de la ciencia ya que se requiere una diversidad de perfiles para desarrollar proyectos en contextos diferentes. La oferta mundial de programas para formar divulgadores de la ciencia se compone de una cantidad considerable de cursos y talleres introductorios y especializados; cursos de capacitación para mediadores o guías de museos; cursos para periodistas; diplomados, especializaciones y posgrados [Reynoso Haynes, 2008]. Algunas de las fórmulas que se han visto en estos cursos para formar 
profesionales en divulgación de la ciencia son las siguientes

[Reynoso Haynes, 2008]:

- Énfasis en los contenidos científicos: Los temarios incluyen temas actuales, polémicos u "obligados", como cambio climático, transgénicos y salud.

- Énfasis en el medio: Proporcionan bases teóricas y prácticas en algún medio, como el escrito, el audiovisual, los museos, los medios masivos y las TICs.

- Énfasis en la teoría: Presentan abordajes teóricos para la divulgación de la ciencia, para lo cual recurren a otras áreas del conocimiento, como la filosofía, la historia, la pedagogía, la literatura, la comunicación y las ciencias sociales.

- Énfasis en la práctica: Se basan en la idea de que lo más importante es la práctica. El alumno adquiere esta práctica participando en el desarrollo de productos o a través de una estancia en un medio de comunicación o instituto de investigación.

- Énfasis en aspectos administrativos y/o de la gestión relacionados con la divulgación de la ciencia: Preparan a sus egresados para laborar en instituciones de investigación o de desarrollo científico y tecnológico.

- Énfasis en la adquisición de habilidades o destrezas complementarias: Algunos ejemplos son el manejo de voz, la expresión corporal y técnicas teatrales para guías de museos.

Por su parte, Massarani, Reynoso-Haynes y col. [2016] publicaron un panorama general de los programas de posgrados, ofrecidos regularmente, que existen en América Latina para formar comunicadores de la ciencia. Se identificaron 22 programas con estas características concentrados en cinco países de los cuales el 65 \% fueron creados en los últimos diez años. Llama la atención el hecho de que los programas reportados en el estudio se concentran solamente en cinco países y en 13 ciudades. Sin embargo, hay que mirar estos números con cuidado pues el artículo fue publicado en 2016 y, debido al carácter emergente del campo, es posible que otros cursos hayan sido creados - como de hecho pasó con nuestro curso de Maestría en Divulgación de la Ciencia, Tecnología y Salud, creado en 2017 en Casa de Oswaldo Cruz, Fundación Oswaldo Cruz - o de lo contrario, algunos otros puede que hayan sido descontinuados.

Las autoras señalan que la experiencia de México en la cual tanto la Dirección General de Divulgación de la Ciencia de la Universidad Nacional Autónoma de México (UNAM) como la Sociedad Mexicana para la Divulgación de la Ciencia y la Técnica (SOMEDICyT) ofrecen diplomados en diferentes regiones del país es un modelo interesante que podría ser adoptado por ejemplo por la RedPOP en la articulación con organizaciones nacionales de divulgación científica, aunque requiere recursos substanciales. Señalan, también, que contar con posgrados virtual o semi-presencial podría servir para llenar la brecha existente, afirmando que hasta la fecha solamente existen cursos cortos en líneas por lo cual crear un diplomado o un posgrado en línea es una tarea pendiente.

Las autoras observaron una gran diversidad en cuanto a requisitos de ingreso, objetivos, contenidos, enfoques, duración y requisitos de egreso de los programas 
de posgrados analizados. Los programas presentan características distintas en cuanto a su contenido, estructura, enfoques, objetivos, duración, perfiles de los alumnos, requisitos de ingreso y egreso y los grados otorgados. Algunas de las fórmulas que se pueden mencionar dan prioridad a diferentes elementos como: los contenidos científicos, algún medio de comunicación, la teoría, la práctica, la adquisición de determinadas destrezas o en aspectos administrativos y/o de gestión. Todos coinciden en su esfuerzo por ofrecer contenidos específicos en el área de la comunicación de la ciencia.

Investigación en divulgación de la ciencia
Guenther y Joubert [2017] realizaron un análisis bibliográfico sistemático de todos los artículos publicados hasta 2016 por las tres principales revistas especializadas a nivel mundial en el área de la divulgación de la ciencia: Science Communication, Public Understanding of Science y JCOM - Journal of Science Communication. El trabajo mostró que, de 57 países donde las instituciones de los autores de los artículos evaluados estaban localizadas, la mayor parte de los artículos publicados en las revistas evaluadas provienen de instituciones ubicadas en América del Norte y en Europa, mientras que pocos provienen de instituciones de Asia y América Latina.

Sin embargo, Rocha y Massarani [2017] demostraron que esta invisibilidad de la producción académica los tres principales periódicos mundiales en la divulgación de la ciencia no corresponde a la realidad. Las autoras realizaron un mapeo de artículos sobre divulgación de la ciencia publicados en periódicos científicos, teniendo como criterio de inclusión en artículos sobre países de América Latina o sobre la región como un todo, pudiendo tener como autores investigadores ligados o no a instituciones latinoamericanas. La recolección de material fue realizada en un período de seis meses, entre marzo y septiembre de 2016, pero se refirió a todos los artículos identificados desde la década de 1980. La búsqueda se realizó en Internet en revistas académicas latinoamericanas y de otras regiones del mundo que publican artículos en el área, incluyendo las tres revistas especializadas en divulgación de la ciencia anteriormente mencionadas (Public Understanding of Science, Science Communication y Journal of Science Communication) o en campos afines que, sistemáticamente, publican artículos en divulgación de la ciencia (por ejemplo, enseñanza de ciencia, comunicación y museología).

El conjunto final de estudios recolectados a lo largo del período contó con 609 artículos académicos publicados por 1.199 autores de 244 instituciones enfocando el tema divulgación de la ciencia en países de América Latina. Esos trabajos fueron publicados en 80 revistas académicas especializadas en el campo de la comunicación pública de la ciencia.

Los artículos también fueron evaluados con relación al año de publicación, que mostró que el estudio más antiguo entre los recolectados fue publicado en 1985. Es importante destacar que la ausencia de artículos publicados anteriormente a ese año se da por una limitación de la metodología del estudio, ya que las autoras no consultaron las versiones impresas de las revistas académicas. Un ejemplo concreto de eso son los diversos artículos sobre divulgación de la ciencia escritos por José Reis, uno de los pioneros de la divulgación de la ciencia en Brasil, por ejemplo en las revistas Ciência e Cultura, de la Sociedade Brasileira para o Progresso da Ciência. A partir de 1997, el número de publicaciones tendió a aumentar, oscilando hasta 2009, cuando comenzó a aumentar constantemente, llegando al número de 99 
artículos publicados en 2014. Teniendo en vista que la etapa de recolección de artículos finalizó en setiembre, el año de 2016 contó con 47 publicaciones.

Un análisis de los países donde las instituciones de los autores están localizadas muestra la gran concentración de publicaciones en Brasil: 509 de los 609 artículos del corpus ( 83 \% del total). A continuación, pero con gran diferencia en relación a Brasil, están Colombia, Argentina y México. Sin embargo, es importante destacar que parte importante de la producción académica de Colombia, Argentina y México, que poseen una capacidad instalada de investigación académica en el área, o incluso de otros, puede (y debe) estar "invisibilizada", por ejemplo, porque la mayor producción es en libros o en revistas impresas no disponibles en Internet.

Solamente 18 de los 609 artículos recolectados son producto de colaboraciones entre países diferentes. De esos, ocho resultan de colaboraciones entre países de América Latina: cuatro entre Brasil y Argentina, dos entre Brasil y Colombia, uno entre Brasil y Venezuela, y uno entre Brasil y México.

Los artículos recolectados fueron agrupados de acuerdo a las categorías que ilustran los temas de investigación en divulgación de la ciencia. Entre ellas, la categoría Medios y ciencia es la que concentra el mayor número de artículos, conteniendo el equivalente a $31 \%$ del número total de los trabajos recolectados, seguida por la categoría Museos y centros de ciencias, con $20 \%$, y Divulgación de la ciencia en la escuela, con $14 \%$.

Consideraciones finales
Con las publicaciones producidas entre 2014 y 2017 bajo el liderazgo de RedPOP y la colaboración de varias organizaciones, es posible tener una visión general de cómo avanza el campo de la divulgación de la ciencia en la región. Por un lado, observamos muchas acciones diversas que están en curso y se realizan por iniciativa de distintos grupos de diferentes países. Pero en muchos casos las acciones no son ofrecidas de forma sistemática, no hay un plan estratégico ni recursos financieros y humanos suficientes. Además, hay todavía muchos sectores de la sociedad no incluidos en las acciones. Por otro lado, identificamos dos áreas importantes que aún están desatendidas: la evaluación del impacto de las acciones en divulgación de la ciencia y la accesibilidad a personas discapacitadas.

Ha sido una grata sorpresa observar que algunos países de la región en cierta medida poseen marcos o legislaciones relacionados con la divulgación de la ciencia que, a diferentes niveles, ha impulsado el sector. Pero en una región de inmensas instabilidades políticas y económicas la divulgación de la ciencia también está en una constante montaña rusa.

La creación de los cursos de posgrados en la región señala la consolidación del área académica en divulgación de la ciencia en la región. Sin embargo, los cursos todavía existen en pocos países (cinco) y están concentrados en pocas ciudades (13). Por lo tanto, consideramos necesaria la creación de una maestría y un doctorado en línea o semi-presencial en español y portugués, que traiga el contexto y las necesidades de la región.

Con respecto a la investigación en divulgación de la ciencia, observamos un incremento importante de artículos producidos en los últimos años. Existe, 
evidentemente, una generación de investigadores en el campo que se consolida y, ojalá, se multiplique. Para ello, es muy importante establecer más colaboraciones entre grupos y países - aún incipiente de acuerdo con los datos recoletados (solamente 18 de los 609 artículos recolectados por Rocha y Massarani [2017] son originados de colaboraciones entre países diferentes) - lo que contribuirá para innovar las metodologías de investigación. También es importante tener revistas académicas en divulgación de la ciencia que permitan ser un foro de intercambio de ideas y de provocaciones intelectuales - y fue justamente por ello que surgió la propuesta de crear JCOM América Latina.

En síntesis, el mensaje de este artículo es: Avanzamos. Pero no lo suficiente. En este contexto, es muy importante sistematizar la iniciativa de impulsar publicaciones que apoyen un diagnóstico constante de la divulgación de la ciencia en América Latina, que permitan comprender mejor cómo está el campo en la región, conocer sus avances pero también sus vacíos y debilidades. Con esto, podemos consolidar estrategias para garantizar un mejor rumbo que incluya a todos los países.

\section{Referencias}

CAMBRE, M. (2015). ‘Museos interactivos de Ciencia y Tecnología en América Latina'. En: RedPOP: 25 años de popularización en América Latina. Ed. por MASSARANI, L. 1. ${ }^{\mathrm{a}}$ ed. Río de Janeiro, Brasil: RedPOP, UNESCO, Museu da Vida, Casa Oswaldo Cruz, Fiocruz, págs. 41-50.

FERNANDEZ, E., BELLO, A. y MASSARANI, L. (2015). ‘Políticas Públicas e Instrumentos para el Desarrollo de la Cultura Científica en América Latina'. En: RedPOP: 25 años de popularización en América Latina. Ed. por MASSARANI, L. 1. ${ }^{\mathrm{a}}$ ed. Río de Janeiro, Brasil: RedPOP, UNESCO, Museu da Vida, Casa Oswaldo Cruz, Fiocruz, págs. 111-131.

- (2016). Políticas públicas e instrumentos para el desarrollo de la cultura científica en América Latina. 1. ${ }^{\mathrm{a}}$ ed. Montevideu, Uruguay: Unesco.

FOG, L. (2004). 'El periodismo científico en Colombia, un lento despegue'. Quark 44 , págs. 59-65.

GUENTHER, L. y JOUBERT, M. (2017). 'Science communication as a field of research: identifying trends, challenges and gaps by analysing research papers'. JCOM 16 (02), A02. https://doi .org/10.22323/2.16020202.

MASSARANI, L., AGUIRRE, C., C., P., REYNOSO, E. y LINDERGAARD, L. (2015). 'RedPOP: 25 años de red en comunicación de la ciencia en América Latina'. En: RedPOP: 25 años de popularización en América Latina. Ed. por MASSARANI, L. 1. ${ }^{\text {a }}$ ed. Río de Janeiro, Brasil: RedPOP, UNESCO, Museu da Vida, Casa Oswaldo Cruz, Fiocruz, págs. 13-26.

MASSARANI, L. y DE CASTRO MOREIRA, I. (2004). 'Divulgación de la ciencia: perspectivas históricas y dilemas permanentes'. Quark 32, págs. 30-35.

- (2016). 'Science communication in Brazil: A historical review and considerations about the current situation'. Anais da Academia Brasileira de Ciências 88 (3), págs. 1577-1595. https://doi .org/10.1590/0001-3765201620150338.

MASSARANI, L., REYNOSO-HAYNES, E., MURRIELLO, S. y CASTILLO, A. (2016). 'Posgrado en Comunicación de la Ciencia en América Latina: un mapa y algunas reflexiones'. [Spanish version of Science Communication Postgraduate Studies in Latin America: a map and some food for thought]. JCOM 15 (05), A03. https://doi.org/10.22323/2.15050203. 
MOREIRA, I. y MASSARANI, L. (2002). 'Aspectos históricos da divulgação científica no Brasil'. En: Ciência e Público: caminhos da divulgação científica no Brasil. Ed. por MASSARANI, L., MOREIRA, I. y BRITTO, F. Rio de Janeiro, Brazil: Casa da Ciência - Centro Cultural de Ciência e Tecnologia da UFRJ, págs. 43-64.

NORBERTO ROCHA, J., MASSARANI, L., GONÇALVEZ, J., FERREIRA, F., ABREU, W., MOLENZANI, A. e INACIO, L. G. (2017). Guía de museos y centros de ciencias accesibles de América Latina y el Caribe. $1 .^{\mathrm{a}}$ ed. Rio de Janeiro, Brasil: RedPOP-UNESCO e Museu da Vida. URL: http://www.redpop . org/guia-de-museos-de-ciencia-accesibles-en-america-latina/ (visitado 22 de agosto de 2018).

PATIÑO, M. L., PADILLA, J. y MASSARANI, L. (2017). Diagnóstico de la Divulgación de la Ciencia en América Latina: Una mirada a la práctica en el campo. 1. ${ }^{\mathrm{a}}$ ed. Ciudad de México, México: Fibonacci e RedPOP.

URL: http://www .redpop.org/wp-content/uploads/2017/06/Diagnostico-d ivulgacion-ciencia_web.pdf (visitado 22 de agosto de 2018).

POLINO, C. (2015). ‘Las encuestas de percepción pública de la ciencia en América Latina: estructura, evolución y comparabilidad'. En: RedPOP: 25 años de popularización en América Latina. Ed. por MASSARANI, L. 1. ${ }^{a}$ ed. Río de Janeiro, Brasil: RedPOP, UNESCO, Museu da Vida, Casa Oswaldo Cruz, Fiocruz, págs. 97-109.

REYNOSO HAYNES, E. (2008). ‘La comunicación de la ciencia y la evaluación de programas para formar comunicadores'. En: Evaluando la comunicación de la ciencia: una perspectiva latinoamericana. Ed. por LOZANO, M. y SÁNCHEZ-MORA, C. Ciudad de México, México: CYTED, UNAM, págs. 193-206.

REYNOSO HAYNES, E., MONTERROSA, C. y MACÍAS, P. (2015). 'La formación de comunicadores de la ciencia en América Latina'. En: RedPOP: 25 años de popularización en América Latina. Ed. por MASSARANI, L. 1. ${ }^{\text {a }}$ ed. Río de Janeiro, Brasil: RedPOP, UNESCO, Museu da Vida, Casa Oswaldo Cruz, Fiocruz, págs. 141-150.

ROCHA, M. y MASSARANI, L. (2017). 'Panorama general de la investigación en divulgación de la ciencia en América Latina'. En: Aproximaciones a la investigación en divulgación de la ciencia en América Latina a partir de sus artículos académicos. Ed. por MASSARANI, L., ROCHA, M., PEDERSOLI, C., ALMEIDA, C., AMORIM, L., CAMBRE, M., NEPOTE, A. C.,

NOBERTO ROCHA, J., AGUIRRE, C., GONÇALVEZ, J. C., CORDIOLI, L. y FERREIRA, F. $1 .^{\text {a }}$ ed. Rio de Janeiro, Brazil: RedPOP e Casa de Oswaldo Cruz, págs. 13-38.

ROCHA, M., MASSARANI, L. y PEDERSOLI, C. (2017). 'La divulgación de la ciencia en América Latina: términos, definiciones y campo académico'. En: Aproximaciones a la investigación en divulgación de la ciencia en América Latina a partir de sus artículos académicos. Ed. por MASSARANI, L., ROCHA, M., PEDERSOLI, C., ALMEIDA, C., AMORIM, L., CAMBRE, M., NEPOTE, A. C., NOBERTO ROCHA, J., AGUIRRE, C., GONÇALVEZ, J. C., CORDIOLI, L. y FERREIRA, F. $1 .^{\text {a }}$ ed. Rio de Janeiro, Brazil: RedPOP e Casa de Oswaldo Cruz, págs. 39-58. 
SÁNCHEZ-MORA, C., REYNOSO-HAYNES, E., SÁNCHEZ MORA, A. M. y TAGÜEÑA, J. (2015). 'Public communication of science in Mexico: past, present and future of a profession'. Public Understanding of Science 24 (1), págs. 38-52. https://doi .org/10.1177/0963662514527204. PMID: 24789844.

TRENCH, B. y BUCCHI, M. (2010). 'Science communication, an emerging discipline'. JCOM 09 (03), C03. https ://doi.org/10.22323/2.09030303.

Autor

Luisa Massarani. Coordina el Instituto Nacional de Comunicación Pública de la Ciencia y Tecnología, con sede en la Fundación Oswaldo Cruz (Fiocruz), y la Maestría en Divulgación de la Ciencia, Tecnología y Salud de Casa de Oswaldo Cruz/Fiocruz. Miembro del Comití Científico de la PCST Network. Coordinadora de SciDev.Net/América Latina y el Caribe (http:/ / www.scidev.net). Premio Mercosur de Ciencia y Tecnología 2014. Premio José Reis de Divulgación Científica 2016. Segundo lugar del Premio Jabuti 2017 de literatura, con el libro infantil de divulgación científica "Se eu fosse..." E-mail: luisa.massarani6@gmail.com. Latina'. JCOM - América Latina 01 (01), A01. https:/ / doi.org/10.22323/3.01010201. 